\title{
Tivantinib (ARQ 197) affects the apoptotic and proliferative machinery downstream of C-MET: role of $\mathrm{Mcl}-1, \mathrm{Bcl}-\mathrm{xl}$ and Cyclin B1
}

\author{
Shuai Lu' ${ }^{1}$, Helga-Paula Török ${ }^{1}$, Eike Gallmeier ${ }^{2}$, Frank T. Kolligs ${ }^{1,3}$, Antonia Rizzani ${ }^{1}$, \\ Sabrina Arena ${ }^{4,5}$, Burkhard Göke ${ }^{1}$, Alexander L. Gerbes ${ }^{1}$, Enrico N. De Toni ${ }^{1}$ \\ ${ }^{1}$ Medizinische Klinik und Poliklinik 2, Klinikum der Universität München, Campus Grosshadern, Munich, Germany \\ ${ }^{2}$ Department of Gastroenterology, Endocrinology and Metabolism, University Hospital of Marburg, Philipps-University of \\ Marburg, Marburg, Germany \\ ${ }^{3}$ Department of Internal Medicine and Gastroenterology, HELIOS Klinikum Berlin-Buch, Berlin, Germany \\ ${ }^{4}$ Department of Oncology, University of Torino, Candiolo, Torino, Italy \\ ${ }^{5}$ Candiolo Cancer Institute-FPO, IRCCS, Candiolo, Italy \\ Correspondence to:
}

Enrico De Toni, e-mail: enrico.detoni@med.uni-muenchen.de

Keywords: HCC, targeted therapies, C-MET, apoptosis

Received: January 28, $2015 \quad$ Accepted: May 28, $2015 \quad$ Published: June 10, 2015

\section{ABSTRACT}

Tivantinib, a C-MET inhibitor, is investigated as a second-line treatment of HCC. It was shown that c-MET overexpression predicts its efficacy. Therefore, a phase-3 trial of tivantinib has been initiated to recruit "c-MET-high"patients only. However, recent evidence indicates that the anticancer activity of tivantinib is not due to c-MET inhibition, suggesting that c-MET is a predictor of response to this compound rather than its actual target. By assessing the mechanisms underlying the anticancer properties of tivantinib we showed that this agent causes apoptosis and cell cycle arrest by inhibiting the anti-apoptotic molecules $\mathrm{Mcl}-1$ and $\mathrm{Bcl}-\mathrm{xl}$, and by increasing Cyclin B1 expression regardless of C-MET status. However, we found that tivantinib might antagonize the antiapoptotic effects of C-MET activation since HGF enhanced the expression of Mcl-1 and Bcl-xl. In summary, we show that the activity of tivantinib is independent of C-MET and describe Mcl-1, Bcl-xl and Cyclin B1 as effectors of its antineoplastic effects in HCC cells. We suggest that the predictive effect of C-MET expression in part reflects the c-MET-driven overexpression of Mcl-1 and Bcl-xl in c-MET-high patients and that these molecules are considered as possible response predictors.

\section{INTRODUCTION}

Tivantinib (ARQ 197) is being investigated in clinical trials as selective orally available c-MET inhibitor [1]. Administered as a single agent in a second-line treatment setting, tivantinib was shown to prolong significantly the time to progression and the survival of hepatocellular carcinoma (HCC) patients in a randomized, placebo-controlled, phase 2 clinical trial $[2,3]$. Analysis of c-MET expression showed a clear advantage in overall survival in patients bearing tumors exhibiting a highly positive staining for c-MET but not in "MET-low" patients. The administration of tivantinib is thus regarded as a promising bona fide biomarker-based therapy of HCC. Consequently, patients' selection in the ongoing phase 3 METIV-HCC trial of tivantinib is based on the detection of high expression of c-MET in tumor biopsies. The predictive value of c-MET in determining survival improvement in patients on tivaninib was recently confirmed by a subgroup analysis of a randomized controlled trial in patients with non-squamous, non-small cell lung cancer $[4,5]$. Although the clinical efficacy of tivantinib in c-MET-high patients in the two aforementioned trials suggests that its anticancer activity is determined by its capability to inhibit c-MET, several in vitro studies published very recently challenged this notion by showing that this compound exerts a remarkable cytotoxic effect in several cell lines without affecting the kinase activity of this receptor. These studies questioned the rationale for the use of this compound in c-MET-high 
patients [6-9] and raised the issue of whether c-MET represents a response predictor of tivantinib rather than its actual target $[10,11]$. In spite of the clinical relevance of this issue, the mechanisms of action of tivantinib as well as those determining the predictive value of c-MET expression still remain to be elucidated.

In the attempt to provide an answer to this question, we decided to investigate the so far still unclear intracellular mechanisms of action of tivantinib on cell death and cell cycle progression, and to assess how their regulation is influenced by this compound in cell lines exhibiting different c-MET expression status $[12,13]$.

\section{RESULTS}

\section{Tivantinib causes a strong loss of cell viability and of colony forming capability in a wide panel of cell lines from gastrointestinal tumors}

The effect of tivantinib on cell viability was assessed in a wide panel of cell lines exhibiting different levels of c-MET expression including 4 HCC cell lines (Fig. 1A), one cholangiocellular carcinoma cell line, and three additional cancer cell lines from tumors of gastrointestinal origin (Fig. S1). Tivantinib caused a dose dependent loss of cell viability with $\mathrm{IC}_{50}$ values comprised between $9.9 \mathrm{nM}$ (Huh7) and $448 \mathrm{nM}$ (Hep3B). These results were clearly confirmed by colony forming assays showing a reduction in the number and size of colonies in cells treated with tivantinib (Fig. 1B, Fig. S1B). As shown in Figure $1 \mathrm{C}-1 \mathrm{D}$, the effect of tivantinib on phosphorylated c-MET was not evident in unstimulated cells due to low basal level of p-c-MET; however, administration of tivantinib with the c-MET ligand HGF caused a decrement of total c-MET as well as of its phosphorylated form in Huh7 or HepG2 cells (Fig. 1D). This phenomenon, which was also reported previously [6], shows that the effect of tivantinib on overall c-MET largely accounts for the observed decrease of c-MET phosphorylation.

\section{Tivantinib enhances apoptosis by inhibiting the mitochondrial regulators of apoptosis Mcl-1 and Bcl-xl}

To assess the mechanisms underlying the decrease in cell viability caused by tivantinib, we subsequently investigated its effect on apoptosis. As shown by the increasing sub-G1 cell fraction at FACS analysis after PI staining (Fig. 2A, S2A) tivantinib caused a dose- and timedependent increase of apoptosis. Induction of apoptosis was observable at the concentration of $533 \mathrm{nM}$ and most cells showed features of apoptosis at a concentration of $1.6 \mu \mathrm{M}$ after 48 hours of incubation (Fig. 2A) with chromatin condensation and nuclear fragmentation at Hoechst staining (Fig. 2C). Accordingly, progressive time- and dose-dependent increase of caspase 3 cleavage (Fig. 2D,
S2B, S3A), increased caspase 3/7 activation (Fig. 2E, S3B) and cleavage of PARP (Fig. S3) were observed.

To further investigate the mechanisms underlying tivantinib-induced apoptosis, the effects of tivantinib on the two major pro-apoptotic signaling pathways-the extrinsic and the intrinsic apoptotic pathways-were assessed. Analysis of Caspase 8 showed a time and dose-dependent cleavage of Pro-caspase 8 and Bid (Fig. 2F), indicating an increased activation of the receptor-mediated apoptotic pathway. Accordingly, administration of tivantinib sensitized Huh7 and HepG2 cells to the action of the TRAIL-R2 agonistic antibody tigatuzumab, which initiates the extrinsic apoptotic pathway dependently on Caspase 8 recruitment [14] (Fig. S4).

Analysis of the activation of the intrinsic apoptotic pathway showed that tivantinib additionally causes the downregulation of the antiapoptotic molecules Bcl-xl and Mcl-1 (Fig. 2F).

To assess the respective functional relevance of the extrinsic and intrinsic pathway activation in determining the effect of tivantinib, Huh7 cells were co-incubated with tivantinib and the Caspase 8 inhibitor Z-IETDFMK; as shown in Fig. 3A, inhibition of Caspase 8 only marginally reduced the proapoptotic effect of tivantinib. In contrast, silencing of Mcl-1 and Bcl-xl by simultaneous co-transfection of specific siRNA sequences in Huh7 cells led to a progressive increase of apoptosis and consequent loss of cell viability over 72 hours (Fig. 3B-3D). Analysis of cell cycle distribution in these samples showed no cell cycle arrest in consequence of Mcl-1 and Bcl-xl silencing (data not shown).

Due to the negligible effect of Caspase 8 inhibition on apoptosis, these data indicate that tivatinib induces apoptosis by shifting the balance between the pro- and anti-apoptotic mitochondrial regulators of the intrinsic signaling pathway (Fig. 2F).

\section{Tivantinib-mediated accumulation of Cyclin B1 is associated with a $\mathbf{G 2} / \mathrm{M}$ cell cycle arrest}

Since tivantinib was reported to induce a G2-cell cycle arrest, we subsequently performed an analysis of the cell fraction in different phases of the cell cycle by FACS (Fig. 4A). Administration of tivantinib caused a G2/M cell cycle arrest in all cell lines assessed, with a corresponding decrease of the fraction of cells in G1 phase. For example, the fraction of cells in $\mathrm{G} 1, \mathrm{~S}$ and $\mathrm{G} 2 / \mathrm{M}$ phase in vehicle treated Huh7 cells were $57.9 \pm 4.1 \%, 10.4 \pm 5.9 \%$, and $31.7 \pm 9.8 \%$ respectively. After $24 \mathrm{~h}$ incubation with tivantinib at the concentration of $1.6 \mu \mathrm{M}$ the fractions of cells in the different phases of cell cycle were $24.1 \% \pm 2.8$, $11.1 \pm 2.0 \%, 64.9 \pm 9.8 \%$ respectively $(P<0.01-$ Fig. 4 A $)$. Similar effects were observed in several cell lines from colon cancer, cholangiocellular and pancreatic cancer cells (data not shown). To determine the mechanisms underlying the $\mathrm{G} 2 / \mathrm{M}$ cell cycle arrest caused by tivantinib, expression of Cyclin B1, which is known to regulate 

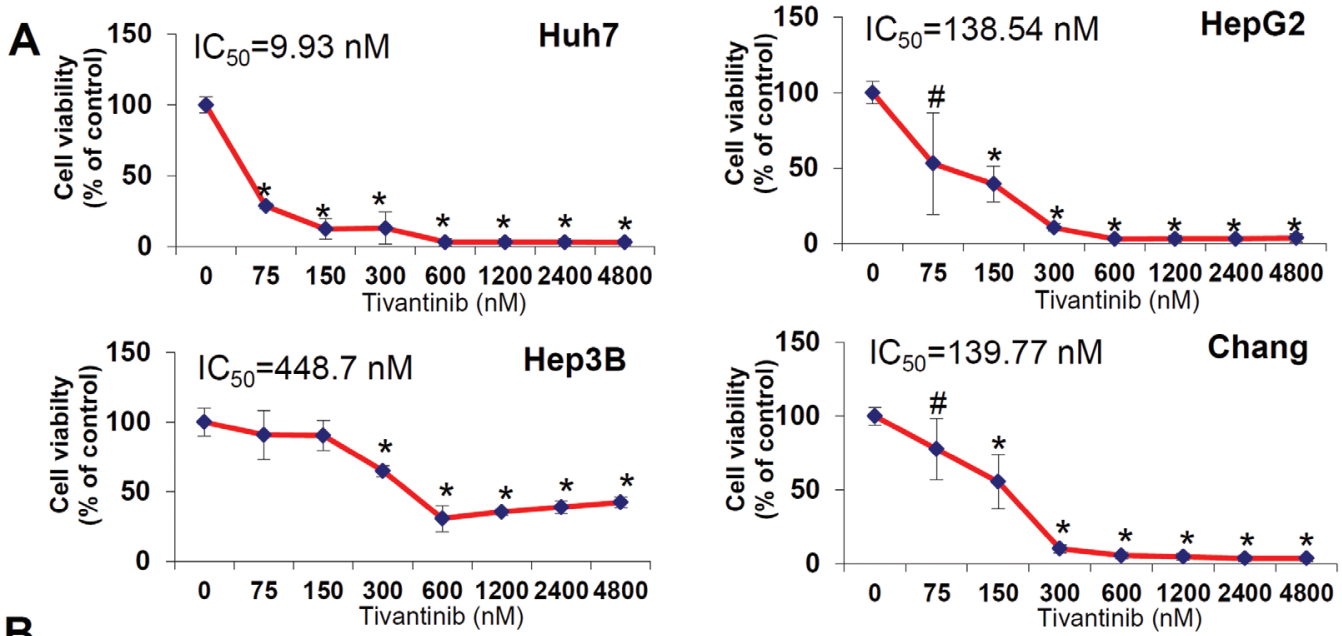

B
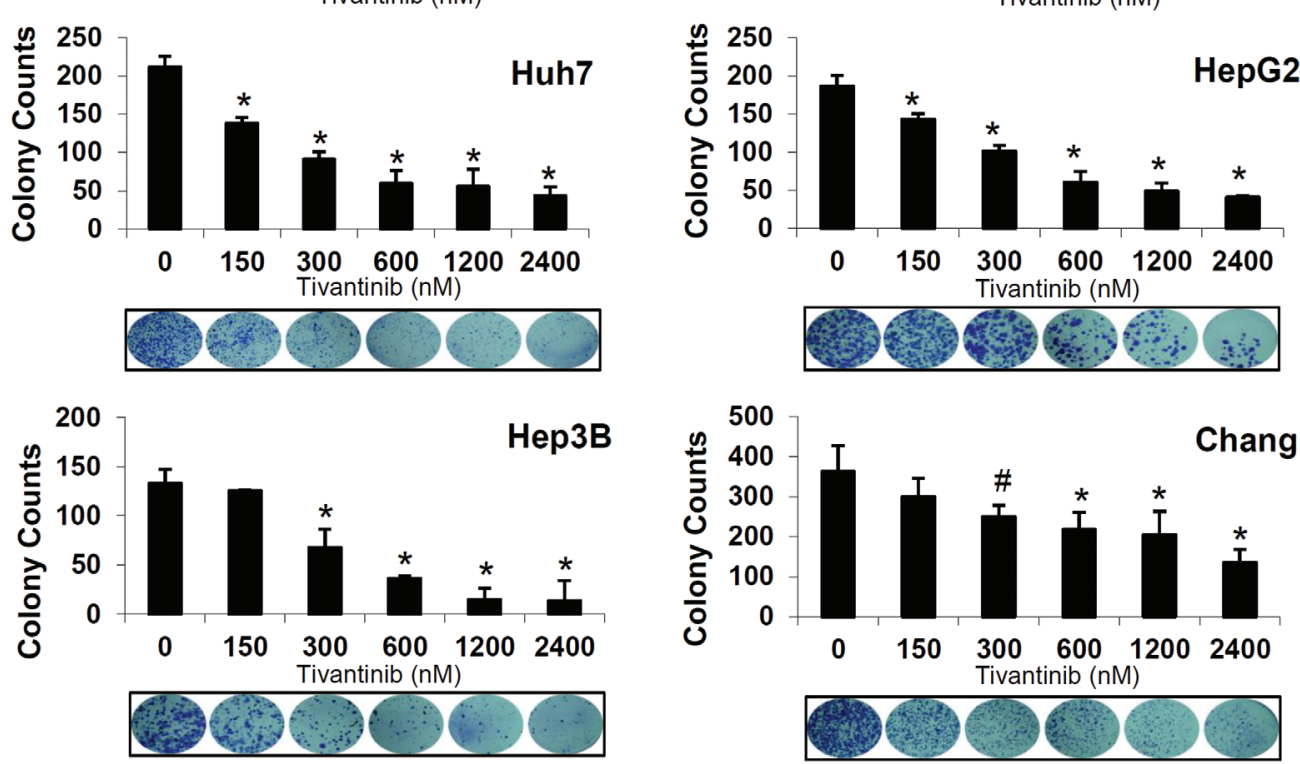

C

Huh7 HepG2 Hep3B Chang

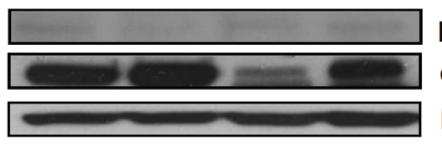

p-MET (Try1234/5)

C-MET

$\beta$-actin

D

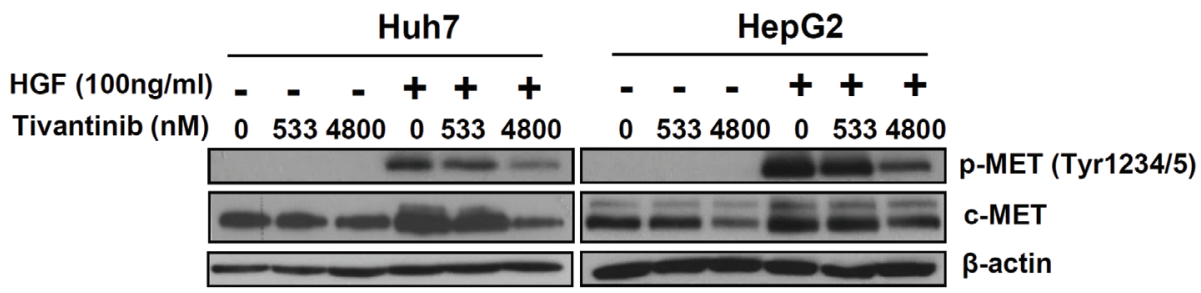

Figure 1: Tivantinib reduces cell viability and colony formation of HCC cells. A. Effect of increasing concentrations of tivantinib on cell viability as judged by Sybr green assays in the indicated cell lines. B. Colony forming assay showing number of colonies and representative figures of the indicated cell lines. Results are expressed as mean and standard deviation of 3 independent experiments each conducted in triplicate. ${ }^{*} p<0.01 ; \# p<0.05$ vs. control treated cells. C. Western blot analysis of c-MET and p-c-MET in the indicated cell lines. D. Effect of tivantinib on phosphorylation status of c-MET in Huh7 and HepG2 cells after administration of the c-MET ligand HGF. For this experiment, cells were incubated with tivantinib for 24 hours; HGF was added at the concentration of $100 \mathrm{ng} / \mathrm{ml}$ for 10 minutes before the cells were harvested. 


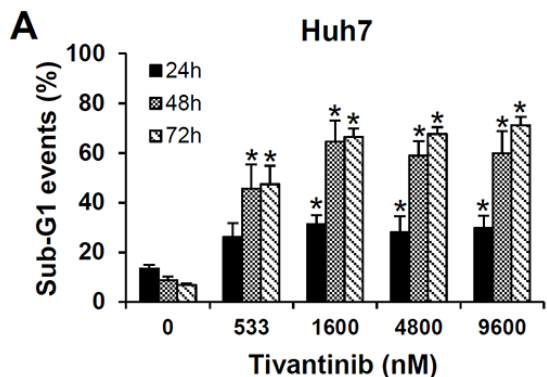

B

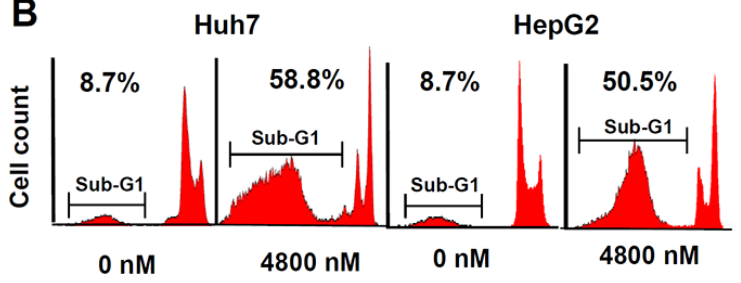

HepG2

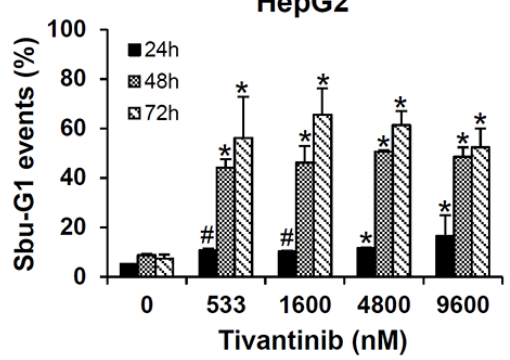

C

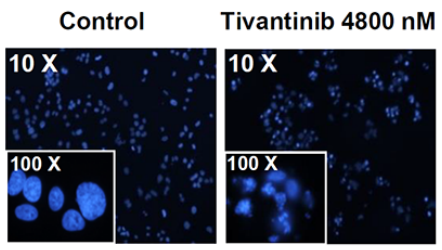

D

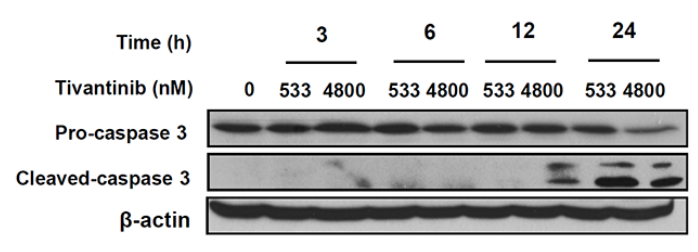

$\mathbf{F}$

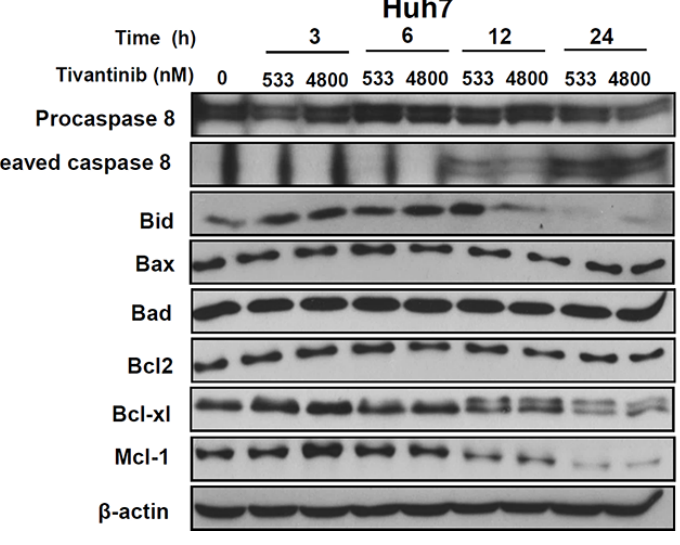

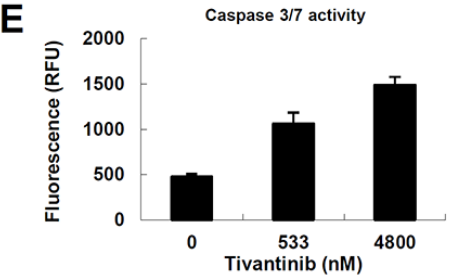

HepG2

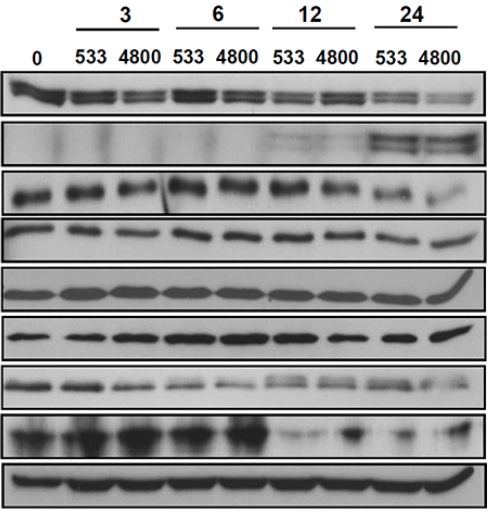

Figure 2: Tivantinib causes apoptosis by activating the mitochondrial apoptotic pathway. A and B. Quantification (A) and typical FACS patterns (B) of apoptotic cells based on the count of the cell fraction showing subdyploid DNA content (sub-G1 fraction) after propidium iodide staining. ${ }^{*} p<0.01 ; \# p<0.05$ in comparison to control treated cells. C. Fluorescence microscopy features after Hoechst staining showing typical chromatin condensation and nuclear fragmentation of Huh7 cells. D and E. Assessment of caspase 3 cleavage by western blot (D) and of caspase 3/7 activation as determined by fluorimetric assessment in HepG2 cells after 24 hours (E). F. Time kinetic of the expression of different regulators of apoptosis was performed by incubating Huh7 or HepG2 cell lines for the indicated time.

cellular transition at the G2/M checkpoint [15], was assessed. Western blot analysis showed a strong increase of Cyclin B1 as soon as 3 hours after incubation (Fig. 4B). To assess whether increase of Cyclin B1 is functionally related to the cycle arrest caused by tivantinib, cells were transfected by specific Cyclin B1-targeting siRNA. As shown in Fig. 4C, silencing of Cyclin B1 prevented the G2/M cell cycle arrest (Fig. 4D) and significantly reduced the loss of cell viability caused by tivantinib (Fig. 4E). These data indicate that a Cyclin-B1 dependent G2/M cell cycle arrest concurs with the induction of apoptosis to determine the antineoplastic effect of tivantinib. 
A

\section{Huh7}

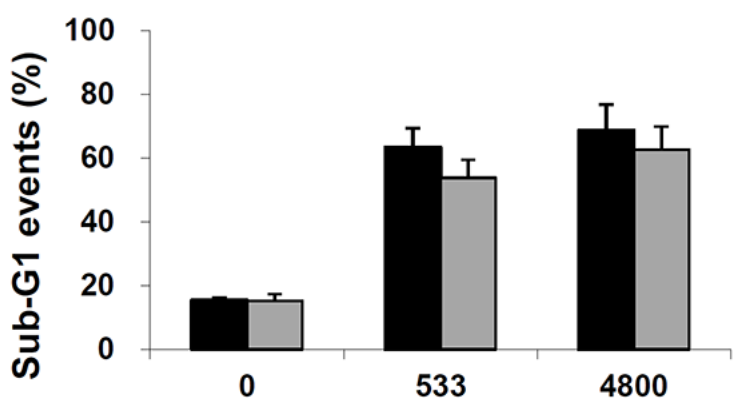

Control

口Caspase-8 Inhibitor Tivantinib (nM)

B

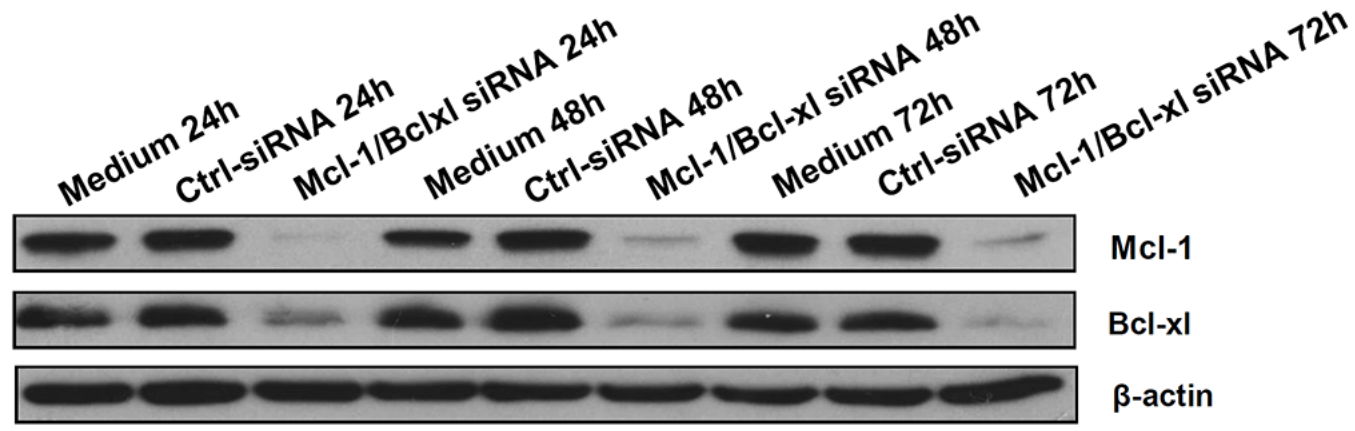

C
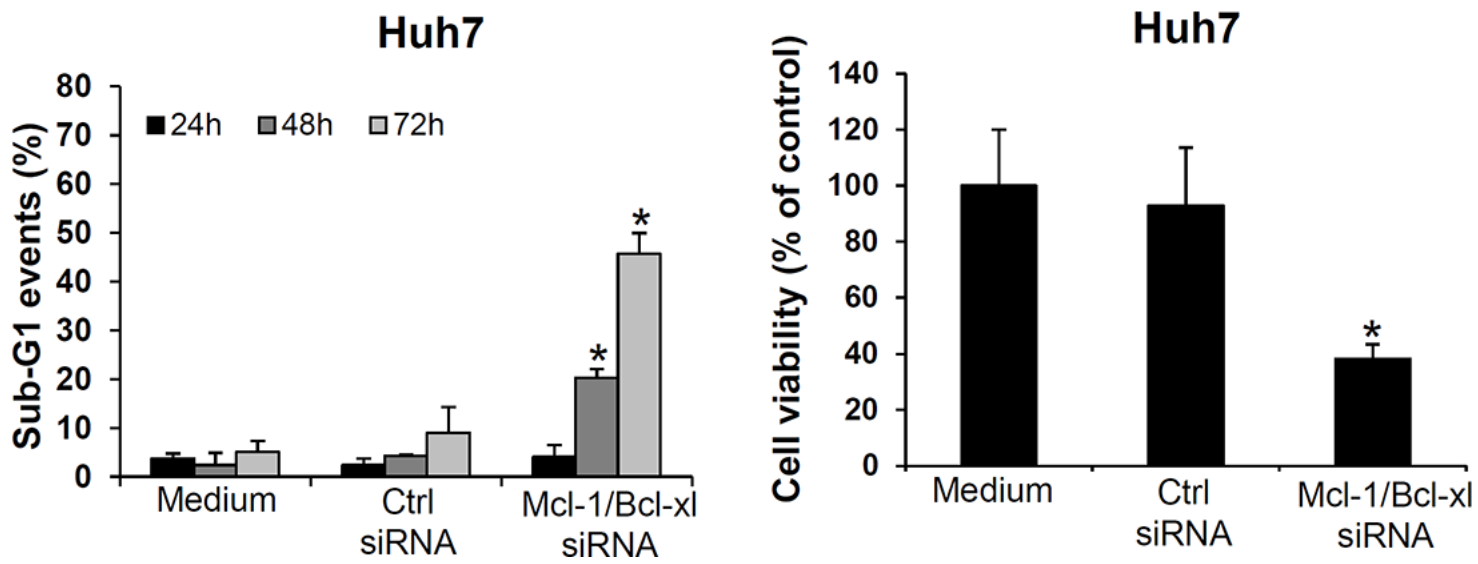

Figure 3: Mcl-1 and Bcl-xl play a functional role in determining the apoptotic effect of tivantinib. A. Effect of Caspase 8 inhibition on tivantinib-mediated apoptosis. FACS analysis was performed to assess nuclear fragmentation 72 hours after administration of tivantinib in the presence or absence of the Caspase8 inhibitor Z-IETD-FMK. B. Effect of Mcl-1 and Bcl-xl silencing by co-transfection of specific siRNA oligonucleotide sequences in Huh7 cells on expression of Bcl-xl and Mcl-1 (each at the concentration of $50 \mathrm{nM}$ ); Medium or non-coding siRNA (Ctrl-siRNA) were used as control. C and D. FACS analysis of apoptosis (C), and cell viability (D) after transfection of siRNA targeting Mcl-1 and Bcl-xl. * $p<0.01$, in comparison to cells transfected with non-coding siRNA. 

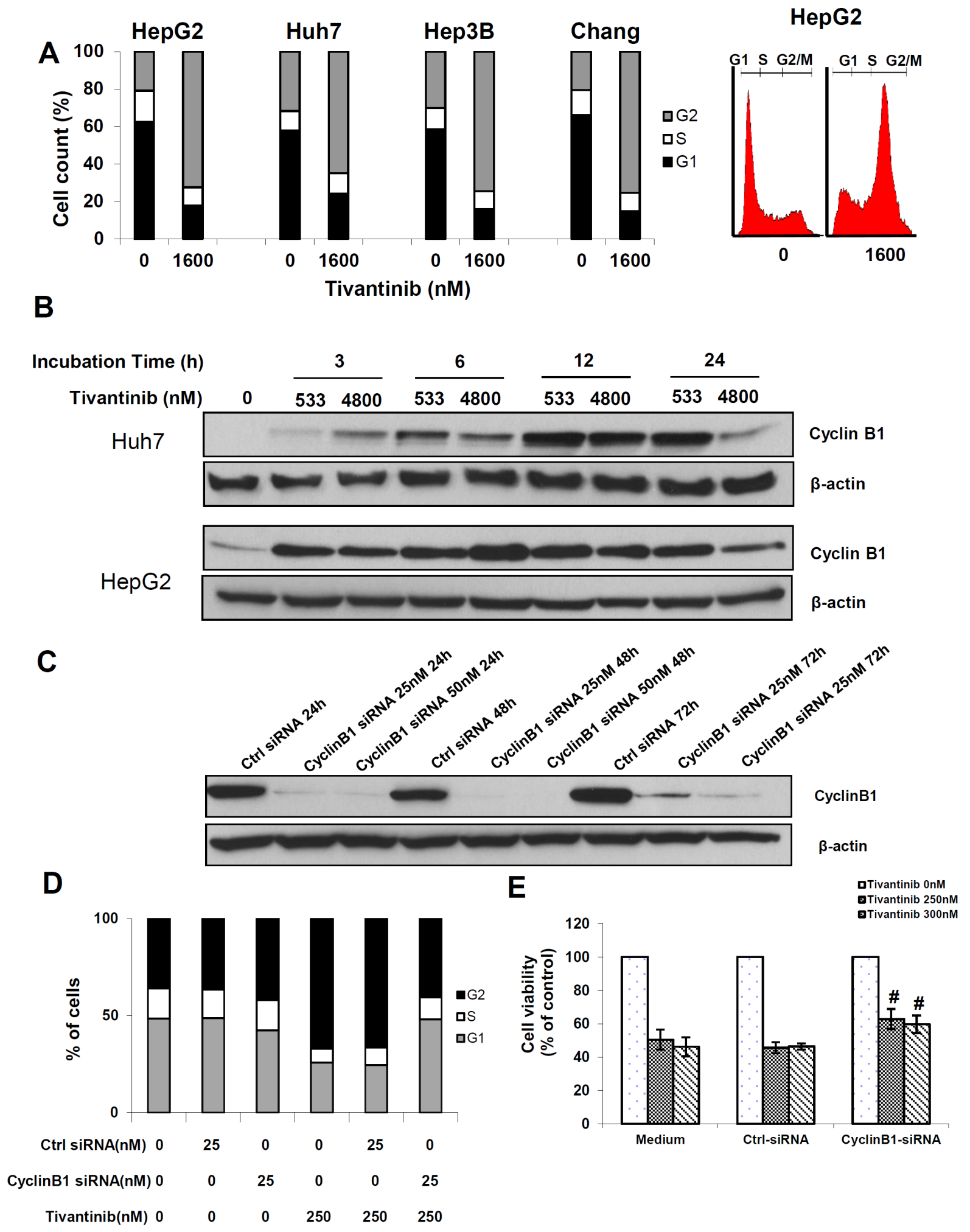

Figure 4: Tivantinib causes a Cyclin B1-dependent G2 cell cycle arrest. A. Cell cycle quantification and typical flow cytometry pattern of G2/M cell cycle arrest of the indicated HCC cell lines after incubation with tivantinib for 24 hours and PI staining. B. Time kinetic of Cyclin B1 activation. C. Effect of Cyclin B1 silencing by specific siRNA (Cyclin B1-siRNA) on Huh7 cells. Non-coding siRNA (Ctrl-siRNA) was used as control. D and E. Effect of Cyclin B1 silencing on cell cycle (D) and cell viability (E) of Huh7 cells. After overnight incubation, cells were transfected with Cyclin B1-targeting siRNA for $24 \mathrm{~h}$, and then treated with tivantinib for 1 hour before harvesting. Cellular viability was assessed by MTS assay in Huh7 cells after 48 hours. \#p $<0.05$ vs. cell incubated in medium only or non-coding siRNA. 


\section{The antiproliferative effect of tivantinib is independent of c-MET but affects targets downstream of c-MET}

After describing Bcl-xl, Mcl-1 and Cyclin B1 as functional targets of tivantinib in determining apoptosis and cell cycle arrest, we assessed whether the influence of tivantinib on these molecules is dependent on its efficacy as inhibitor of c-MET, as the predictive significance of this receptor in clinical trials seems to suggest $[2,16]$. To this aim, we assessed the effects of c-MET silencing by siRNA transfection in Huh7 cells; additionally, we compared the effect of tivantinib on DLD1 cells, which express the native form of c-MET vs. that on syngenic DLD1 c-MET exon $16 \mathrm{KO}$ cell lines, which express a genetically modified variant of c-MET lacking the binding site of tivantinib. Silencing of c-MET by siRNA failed to increase apoptosis and cell cycle arrest in Huh7 cells and only modestly affected cell viability in these cells (Fig. 5A-5D). We also observed that the effect of tivantinb on cell viability, cell cycle and apoptosis in DLD1 wild type (WT) cells was indistinguishable from that observed in two independent c-MET exon 16 knock out cell clones (KO1 and KO2) (Fig. 5E-5G). Western blot analysis of these DLD1 cell clones showed that the expression of Mcl-1 and Bcl-xl was reduced and that of
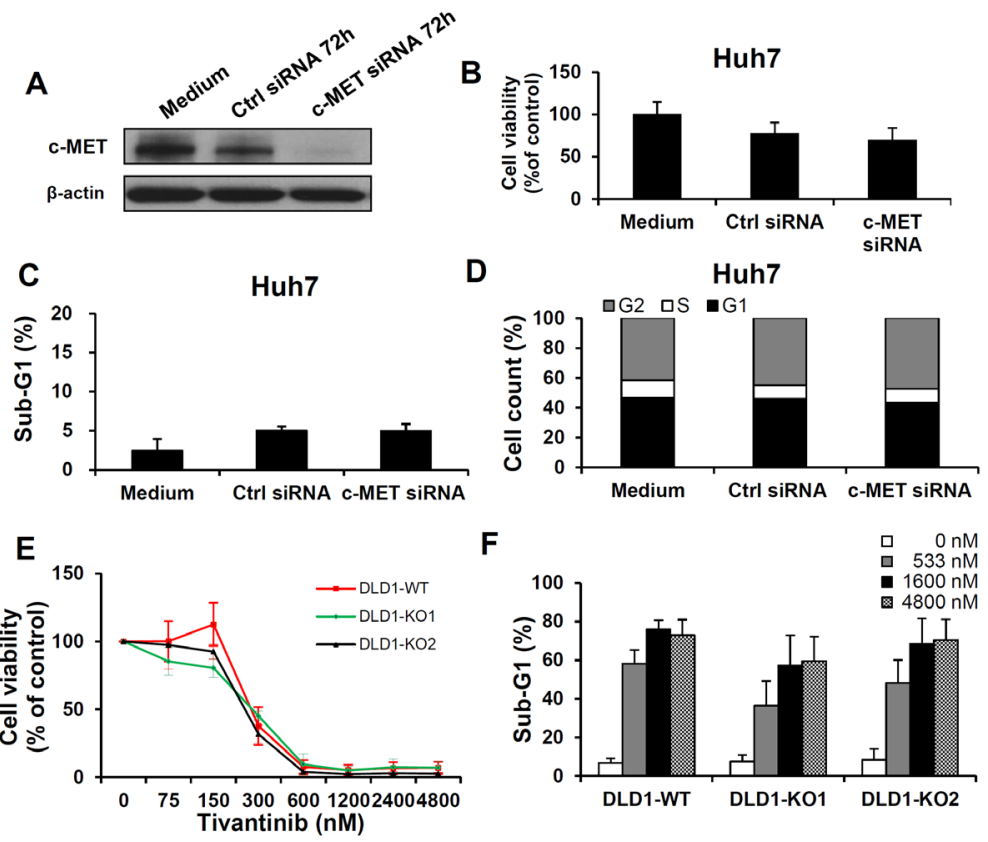

G
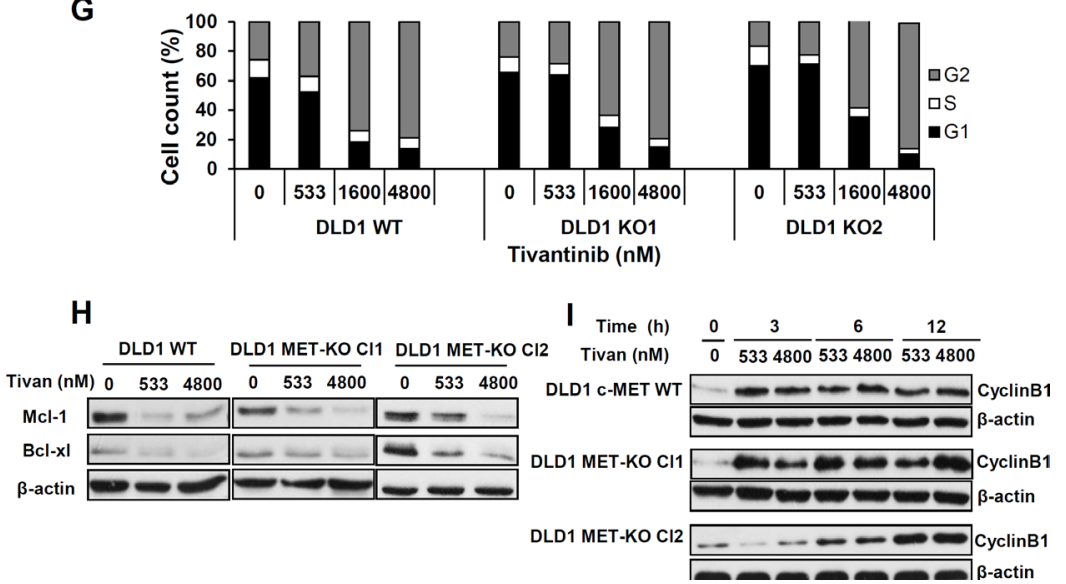

Figure 5: The antineoplastic effect of tivantinib is not dependent on c-MET. A, B, C and D. Effect of c-MET silencing on the expression of c-MET (A) on cell viability (B), apoptosis (C) and cell cycle (D), in Huh7 cells 72 hours after transfection of specific (c-METsiRNA) or non-coding siRNA (ctrl-siRNA). E, F, G, H and I. Effect of tivantinib on $c$-MET wild-type DLD1 colorectal cancer cell lines or in $c-M E T$ exon 16 knock-out DLD1 (two different clones) as determined by viability assay 6 days after incubation with tivantinib (E), assessment of apoptosis (F) and cell cycle analysis (G) 48 hours after incubation with tivantinib. Western Blot analysis (H) of Mcl-1 and Bcl-xl after 24 hours incubation with tivantinib andtime kinetic of Cyclin B1 activation (I) in these cell lines. 
Cyclin B1 increased in both wild type and c-MET-exon 16 knock out DLD1 cells (Fig. 5H and 5I) upon incubation with tivantinib. The biological effects of tivantinb and the associated molecular changes observed in Huh7 and HepG2 cell lines were thus reproducible in 2 additional cell clones derived from DLD1 colorectal cancer cells and were independent of the expression of c-MET; this suggests that the antineoplastic activity of tivantinib is largely independent of its effect on this receptor.

We subsequently hypothesized that the significance of c-MET as predictor of the outcome of patients on tivantinib may be related to the fact that Mcl-1, Bcl-xl or Cyclin B1 are downstream targets of c-MET, and that the efficacy of tivantinib in tumors overexpressing c-MET may reflect the effect of this compound on c-MET-driven overexpression of these molecules.

To assess this possibility, a time kinetic of c-MET, Bcl-xl, Mcl-1 and Cyclin B1 expression was performed in Huh7 and HepG2 cells after incubation with HGF, which is the only known ligand of c-MET. This analysis showed that activation of c-MET leads to increased phosphorylation of c-MET (Tyr1349) and to increased Mcl-1 and Bcl-xl. However, a slight increase of Cyclin B1 could also be observed upon c-MET stimulation (Fig. 6). These data show that while tivantinib might antagonize the effect of c-MET-mediated expression of Mcl-1 and Bcl-xl, the effect of tivantinib Cyclin B1 might not be predictable by the expression of c-MET.

\section{DISCUSSION}

c-MET inhibitors are considered a promising therapeutic option for tumours exhibiting c-MET-status alterations [17]. Tivantinib prolonged time-to-progression and overall survival among c-MET diagnostic-high HCC patients as a second line treatment after failure of a first line sorafenib treatment $[2,3]$ Therefore, only patients selected according to a "c-MET-high" status are recruited in the ongoing phase-3 study of tivantinib in HCC [3]. Unexpectedly, the concept of tivantinib as c-MET inhibitor has been recently challenged, and it has been suggested that c-MET might represent a predictor of efficacy rather than the actual target of this compound [6-9]. In this paper we addressed this issue 1) by investigating the so far unknown molecular mechanisms underlying apoptosis and cell cycle induced by tivantinib and 2) by assessing whether these effects are specifically dependent on the effect of tivantinib on c-MET. These topics and their clinical implications are discussed below.

\section{Molecular mechanisms underlying the effect of tivantinib on apoptosis and cell cycle}

Since tivantinib was previously shown to induce apoptosis and G2/M-cell cycle arrest, we first assessed its effect on crucial mechanisms of apoptosis and cell cycle control. Assessment of apoptosis showed that tivantinb causes cleavage of Procaspase- 8 followed by the cleavage of Bid. Cleavage of Caspase 8 is a hallmark of the activation of the extrinsic apoptotic pathway, which is initiated at the cell membrane in consequence of the stimulation of the "death-receptors" such as TRAIL-R1 and-R2 or CD95. Accordingly, administration of tivantinib sensitized several cancer cell lines to the action of the TRAIL-R2 agonistic antibody tigatuzumab (Fig. S4). Nevertheless, inhibition of Caspase 8 only marginally affected apoptosis induced by tivantinib indicating that activation of the extrinsic signalling pathway plays a marginal role in triggering apoptosis observed in our system.

As we assessed the effect of tivantinib on the intrinsic signaling pathway we observed a strong decrease of the antiapoptotic proteins Bcl-xl and Mcl-1. The intrinsic pathway is regulated by the balance between pro-apoptotic molecules (such as Bax, Bak, Bim and Bid) and antiapoptotic molecules (such as Bcl-2, Bcl-xl and $\mathrm{Mcl}-1$ ). Mcl-1 and Bcl-xl are Bcl-2-related proteins which bind to Bax and Bak, thereby blocking their pro-apoptotic interaction with the outer surface of the mitochondria [18]. The functional relevance of Bcl-xl and Mcl-1 in determining apoptosis mediated by tivantinib was confirmed by si-RNA against Bcl-xl and Mcl-1 which caused apoptosis to an extent comparable with that observed upon tivantinib administration. The effect on Mcl-1 of tivantinib on Mcl-1 is in keep with previous findings showing that $\mathrm{CKD}$ inhibitors trigger apoptotic cell death by inhibiting Mcl-1 [19]. These data show that

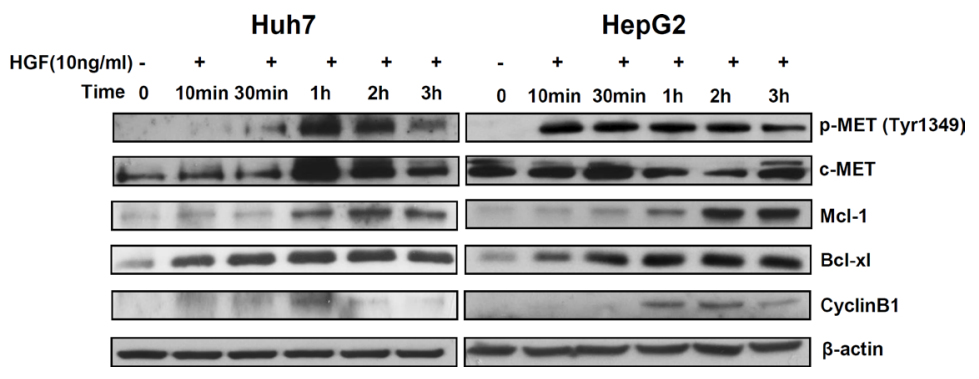

Figure 6: Mcl-1 and Bcl-xl are regulated by stimulation of c-MET. Time kinetic of phospho-MET, Mcl-1, Bcl-xl and Cyclin B1 at western blot analysis. Huh7 and HepG2 cells were incubated with $10 \mathrm{ng} / \mathrm{ml}$ human recombinant HGF and collected at the indicated time points. 
while cleavage of Caspase 8 and activation of the extrinsic apoptotic pathway might play a role in determining the effect of tivantinib in vivo e.g. by affecting the response to endogenous TRAIL, tivantinib causes apoptosis principally by shifting the balance between the mediators of the mitochondrial pathway by inhibiting Bcl-xl and Mcl-1.

In addition, we observed a strong increase of Cyclin $\mathrm{B} 1$ as soon as 3 hours after administration of tivantinib. Cyclin B1 controls the G2-M cell cycle transition [20, 21] and its elevation was shown to cause mitotic delay in Hela cells $[15,22]$. In agreement with these previous reports, we found that silencing of Cyclin B1 reversed the G2/M cell cycle arrest caused by tivantinib and significantly reduced its effect on cell viability.

\section{c-MET expression and response to tivantinib.}

Experiments conducted with $c-M E T$ exon $16 \mathrm{KO}$ DLD1 cell lines, which lack the tivantinib-binding site of c-MET, showed that the effect of tivantinib in these cell lines was indistinguishable from that observed in parentalWild Type DLD1 cell lines. Accordingly, tivantinib led to the downregulation of Bcl-xl, Mcl-1 and to the upregulation of Cyclin B1 independently of the c-MET status of these cell clones. Moreover, silencing of c-MET by specific siRNA failed to reproduce the effect of tivantinib on apoptosis and cell cycle. Administration of tivantinib caused a decrement of total c-MET and, to the same extent, of its phosphorylated form, indicating that the decrease of overall c-MET largely accounts for the observed decrease of c-MET phosphorylation. Nevertheless, our results show that the antineoplastic activity of tivantinib is largely independent of its effect as c-MET inhibitor.

This discrepancy between our data and the predictive effect of c-MET expression in the clinical setting might be due to differences related to the higher complexity of the mechanisms of action of tivantinib in vivo, e.g. those underlying invasion and metastasis formation [23], and to microenvironmental changes related to previous administration of sorafenib in these patients. Such hypothesis would be supported by the reported role of c-MET in hypoxic conditions and in resistance to anti-angiogenic therapy [24, 25]. Nevertheless, the remarkable effect of tivantinib on cell viability, cell cycle and apoptosis observed in our experiments suggests that these effects account for the antineoplastic effects of tivantinib observed in the clinical setting.

To find possible explanations for the predictive relevance of c-MET in clinical trials, we hypothesized that Bcl-xl, Mcl-1 and Cyclin B1 are downstream targets of c-MET, and that the efficacy of tivantinib on tumors overexpressing c-MET may reflect the effect of this compound on c-MET-driven overexpression of these molecules. Stimulation of c-MET by HGF showed a clear increase of Bcl-xl and Mcl-1 suggesting that tivantinib might antagonize the effect of c-MET-dependent expression of these molecules in cells overexpressing this receptor regardless of the inhibitory effect of tivantinib on the kinase activity of c-MET.

Although the mechanisms by which tivantinib causes such molecular alterations still remains elusive, a possible answer to this issue might be provided by the recent works published by Basilico [6], Katayama [7], and their colleagues. These authors found that tivantinib exerted a cytotoxic effect in several cell lines without affecting the function of c-MET by causing microtubule stabilization or impairment of tubulin polymerization, respectively. Interestingly, microtubule-damaging agents are known to increase Cyclin B1 and to activate the spindle assembly checkpoint, and degradation of Mcl-1 has been shown to occur as consequence of administration of microtubule-targeting agents [26]. It is thus likely

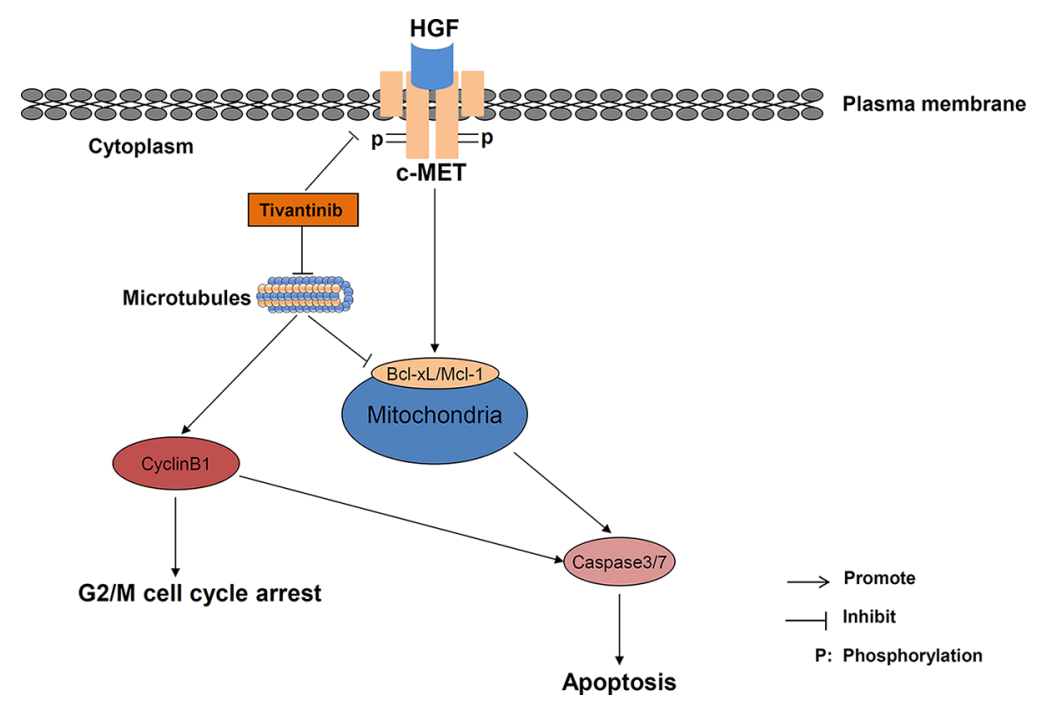

Figure 7: Schematic diagram of the postulated interaction between tivantinib and intracellular mechanisms of apoptosis and cell cycle regulation. 
that tivantinib affects cell proliferation and apoptosis by impinging on microtubule formation and stability (Fig. 7).

In summary, we identified Bcl-xl, Mcl-1 and Cyclin B1 as mediators of the antineoplastic effects of tivantinib. Our study provides a possible explanation for the preferential effect of tivantinib in c-MET-high patients but indicates that the beneficial clinical effect of tivantinib may not be predictable solely by c-MET, and prompts for the evaluation of the expression of other molecules, such as $\mathrm{Mcl}-1, \mathrm{Bcl}-\mathrm{xl}$ and Cyclin B1 as possible and potentially more reliable markers for patients' selection.

\section{MATERIALS AND METHODS}

\section{Cell lines culture and reagents}

Huh7, HepG2, Hep3B, Chang cells (hepatocellular carcinoma), TFK1 (human cholangiocarcinoma), DLD1 cells (human colon carcinoma), PL5 and PANC1 cells (human pancreatic carcinoma) were used for in vitro experiments. DLD1 colorectal cancer cells and two different syngenic $c$-MET exon 16 knock-out DLD-1 clones were provided by A. Bardelli and S. Arena and have been previously described [13]. All cell lines were cultivated in standard cell culture media as recommended by the providers. Tivantinib (ARQ197, provided by ArQule, Woburn MA, USA.) was dissolved in 100\% DMSO and stored at $-20^{\circ} \mathrm{C}$. Tigatuzumab (CS-1008, by Daiichi Sankyo Pharma Development, Edison NJ, USA) was provided as a $10 \mathrm{mg} / \mathrm{ml}$ solution and stored at $4^{\circ} \mathrm{C}$. Recombinant Human HGF was purchased from R\&D (Minneapolis, MN USA).

\section{Cell viability assay}

Cells were seeded onto 96-well plates at different cell densities to avoid overgrowth $\left(0.6-3.5 \times 10^{3} /\right.$ well $)$ and were treated with increasing concentrations of tivantinib or vehicle. To investigate the effect of tivantinib on cell viability at low concentrations, cells were kept in culture for 6 days. At day 6 cells were washed with PBS, underwent

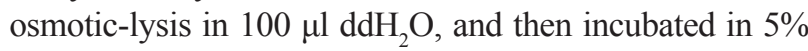
$\mathrm{CO}_{2}$ incubator for 45 minutes. Fluorescence was measured after addition of $0.2 \%$ Sybr green (Cytoflour Series 4000, Applied Biosystems, Framingham, MA USA).

\section{Colony formation assay}

4-5 $\times 10^{3}$ cells were plated onto 6-well plates. After 24-h incubation with tivantinib cells were allowed to grow for 3 weeks. Colony formation was evaluated after the cells were fixed in $9 \%$ paraformaldehyde and stained with crystal violet for 30 minutes (Sigma, St. Louis, MO USA). Total cell colonies in each well were counted after being photographed.

\section{Apoptosis and cell cycle assay}

$8.0 \times 10^{4}-1.5 \times 10^{5}$ cells were seeded in 12 -well plates and treated after overnight incubation. Fluorescence activated cell sorting (FACSAccuri-C6 flow cytometer, BD Biosciences San Jose, CA USA) was performed to detect the sub-G1 cell fraction to determine apoptosis and the respective different phases of cell cycle after propidium iodide (PI) staining as previously described [27]. In addition, apoptosis was assessed morphologically by Hoechst 33342 staining and fluorescence microscopy.

\section{Western blot and caspase activity assays}

Cells growing in Petri dishes were collected and lysed in cell lysis buffer (Cell Signaling Technology, Boston MA USA) containing $20 \mathrm{mmol} / \mathrm{L}$ Tris-HCL (PH7.5), $150 \mathrm{mmol} / \mathrm{L} \mathrm{NaCl}, 1 \mathrm{mmol} / \mathrm{L}$ $\mathrm{Na}_{2}$ EDTA, $1 \mathrm{mmol} / \mathrm{L}$ EGDA, 1\% Triton, $2.5 \mathrm{mmol} / \mathrm{L}$ sodium pyrophosphate, $1 \mathrm{mmol} / \mathrm{L} \beta$-glycerophosphate, $1 \mathrm{mmol} / \mathrm{L} \mathrm{Na}_{3} \mathrm{VO} 4,1 \mu \mathrm{g} / \mathrm{ml}$ leupeptin, and $1 \mathrm{mmol} / 1$ phenylmethane sulphonyl fluoride. Membranes were incubated overnight at $4^{\circ} \mathrm{C}$ with specific antibodies in TBS-T (TBS-0.1\% Tween20) and subsequently for one hour with the appropriate horseradish-peroxidaseconjugated anti-rabbit/mouse secondary antibody. The following mouse monoclonal antibodies were used: Anti-CyclinB1 (Santa Cruz Biotechnology, Dallas Texas USA), anti-Caspase-8, anti- $\beta$-actin (both from Cell Signaling Technology, Boston MA USA). The following rabbit polyclonal antibodies were used: anti-Caspase-3, anti-PARP, anti-Bid, antiBad, anti-Bcl-2, anti-Bcl-xl, anti-Bax, anti-Mcl-1 (all from Cell Signaling Technology), anti-c-MET, antiphosphorylated c-MET (Tyr1349 and Tyr1234/1235). For Caspase-3/7 activity assays the Apo-ONE ${ }^{\circledR}$ Reagent kit (Promega, Madison WI USA) was used according to the instructions of the manufacturer. Actin was used for control of appropriate protein load for each membrane. In figures, one actin loading control has been exemplarily shown.

\section{Small interfering RNA and transfection}

Cyclin B1, Bcl-xl, Mcl-1 and c-MET were silenced by small interfering RNA (siRNA) or non-coding siRNA. Specific siRNA SMARTpool ${ }^{\circledR}$ sequences consist of 4 pooled 21-nucleotide RNA oligonucleotides forming a 19-bp duplex core with 2-nucleotide 3 overhang and were purchased from Dharmacon, Inc. (Thermo Scientific, Rockford IL USA). For transfection the DharmaFECT4 reagent (Thermo Scientific, Rockford IL USA) was used according to the manufacturer's instructions. 


\section{Statistical analysis}

Statistical analysis was performed by using IBM SPSS Statistics (SPSS Inc, IL USA). Comparison of means was performed by two-tailed Student's test. Results are expressed as mean and standard deviation (SD) of at least 3 independent experiments. $P$ values $<0.05$ were regarded as statistically significant.

\section{ACKNOWLEDGMENTS}

We thank Andreas Ziesch for his valuable technical assistance. This study was supported by the Deutsche Forschungsgemeinschaft (DFG) with grants DFG TO 605/2-1 to EDT, by the Else Kröner-Fresenius Stiftung with the grant 2011_A226 to EDT and by the Curt-Bohnewand-Fonds at the Ludwig-Maximilian University, Munich. SL was financed by the China Scholarship Council (CSC) in the context of its exchange program with the University of Munich.

\section{REFERENCES}

1. Bagai R, Fan W, Ma PC. ARQ-197, an oral small-molecule inhibitor of c-Met for the treatment of solid tumors. IDrugs. 2010; 13:404-414.

2. Santoro A, Rimassa L, Borbath I, Daniele B, Salvagni S, Van Laethem JL, Van Vlierberghe H, Trojan J, Kolligs FT, Weiss A, Miles S, Gasbarrini A, Lencioni M, Cicalese L, Sherman M, Gridelli C, et al. Tivantinib for secondline treatment of advanced hepatocellular carcinoma: a randomised, placebo-controlled phase 2 study. Lancet Oncol. 2013; 14:55-63.

3. Trojan J, Zeuzem S. Tivantinib in hepatocellular carcinoma. ExpertOpinInvestigDrugs. 2013; 22:141-147.

4. Scagliotti G, Novello S, Ramlau R, Favaretto A, Barlesi F, Akerley W, Von Pawel J, Shuster D, Schwartz B, Sandler A. MARQUEE: A randomized, double-blind, placebocontrolled, phase 3 trial of tivantinib (ARQ 197) plus erlotinib versus placebo plus erlotinib in previously treated patients with locally advanced or metastatic, non-squamous, non-small-cell lung cancer (NSCLC). Eur J Cancer. 2013; 49:S798-S799.

5. Agwa ES, Ma PC. Targeting the MET receptor tyrosine kinase in non-small cell lung cancer: emerging role of tivantinib. Cancer management and research. 2014; 6:397-404.

6. Basilico C, Pennacchietti S, Vigna E, Chiriaco C, Arena S, Bardelli A, Valdembri D, Serini G, Michieli P. Tivantinib (ARQ197) displays cytotoxic activity that is independent of its ability to bind MET. Clin Cancer Res. 2013; 19:2381-2392.

7. Katayama R, Aoyama A, Yamori T, Qi J, Oh-Hara T, Song Y, Engelman JA, Fujita N. Cytotoxic Activity of Tivantinib (ARQ 197) Is Not Due Solely to c-MET Inhibition. Cancer research. 2013; 73:3087-3096.
8. Calles A, Kwiatkowski N, Cammarata BK, Ercan D, Gray NS, Janne PA. Tivantinib (ARQ 197) efficacy is independent of MET inhibition in non-small-cell lung cancer cell lines. Molecular oncology. 2015; 9:260-269.

9. Aoyama A, Katayama R, Oh-Hara T, Sato S, Okuno Y, Fujita N. Tivantinib (ARQ 197) Exhibits Antitumor Activity by Directly Interacting with Tubulin and Overcomes ABC Transporter-Mediated Drug Resistance. Molecular cancer therapeutics. 2014; 13:2978-2990.

10. Rimassa L, Bruix J, Broggini M, Santoro A. Tivantinib (ARQ197) displays cytotoxic activity that is independent of its ability to bind MET - letter. Clinical cancer research: an official journal of the American Association for Cancer Research. 2013; 19:4290.

11. Michieli P, Di Nicolantonio F. Targeted therapies: Tivantinib-a cytotoxic drug in MET inhibitor's clothes? Nature reviews Clinical oncology. 2013; 10:372-374.

12. Eathiraj S, Palma R, Volckova E, Hirschi M, France DS, Ashwell MA, Chan TC. Discovery of a novel mode of protein kinase inhibition characterized by the mechanism of inhibition of human mesenchymal-epithelial transition factor (c-Met) protein autophosphorylation by ARQ 197. The Journal of biological chemistry. 2011; 286:20666-20676.

13. Arena S, Pisacane A, Mazzone M, Comoglio PM, Bardelli A. Genetic targeting of the kinase activity of the Met receptor in cancer cells. Proceedings of the National Academy of Sciences of the United States of America. 2007; 104:11412-11417.

14. Forero-Torres A, Shah J, Wood T, Posey J, Carlisle R, Copigneaux C, Luo FR, Wojtowicz-Praga S, Percent I, Saleh M. Phase I trial of weekly tigatuzumab, an agonistic humanized monoclonal antibody targeting death receptor 5 (DR5). Cancer BiotherRadiopharm. 2010; 25:13-19.

15. Ito $\mathrm{S}$, Ishii A, Kakusho N, Taniyama C, Yamazaki S, Fukatsu R, Sakaue-Sawano A, Miyawaki A, Masai H. Mechanism of cancer cell death induced by depletion of an essential replication regulator. PLoSOne. 2012; 7:e36372.

16. Eng C, Hart LL, Severtsev A, Gladkov O, Mueller L, Kopp MV, Vladimirov VI, Langdon RM, Kotiv B, Barni S, Hsu C, Bolotin E, Von Roemeling R, Schwartz BE, Bendell JC. A randomized, placebo-controlled, phase I/II study of tivantinib (ARQ 197) in combination with cetuximab and irinotecan in patients (pts) with KRAS wild-type (WT) metastatic colorectal cancer (CRC) who had received previous front-line systemic therapy. J Clin Oncol 31, 2013.

17. Tang C, Fontes Jardim DL, Falchook GS, Hess K, Fu S, Wheler JJ, Zinner RG, Naing A, Tsimberidou AM, De Melo Galgiato D, Westin SN, Meric-Bernstam F, Kurzrock R, Hong DS. MET nucleotide variations and amplification in advanced ovarian cancer: characteristics and outcomes with c-Met inhibitors. Oncoscience. 2014; 1:5-13.

18. Soriano ME, Scorrano L. Traveling Bax and forth from mitochondria to control apoptosis. Cell. 2011; 145:15-17. 
19. Bettayeb K, Baunbaek D, Delehouze C, Loaec N, Hole AJ, Baumli S, Endicott JA, Douc-Rasy S, Benard J, Oumata N, Galons H, Meijer L. CDK Inhibitors Roscovitine and CR8 Trigger Mcl-1 Down-Regulation and Apoptotic Cell Death in Neuroblastoma Cells. Genes \& cancer. 2010; 1:369-380.

20. Soria JC, Jang SJ, Khuri FR, Hassan K, Liu D, Hong WK, Mao L. Overexpression of cyclin B1 in early-stage non-small cell lung cancer and its clinical implication. Cancer Res. 2000; 60:4000-4004.

21. Agarwal R, Gonzalez-Angulo AM, Myhre S, Carey M, Lee JS, Overgaard J, Alsner J, Stemke-Hale K, Lluch A, Neve RM, Kuo WL, Sorlie T, Sahin A, Valero V, Keyomarsi K, Gray JW, et al. Integrative analysis of cyclin protein levels identifies cyclin b1 as a classifier and predictor of outcomes in breast cancer. ClinCancer Res. 2009; 15:3654-3662.

22. Beauman SR, Campos B, Kaetzel MA, Dedman JR. CyclinB1 expression is elevated and mitosis is delayed in HeLa cells expressing autonomous CaMKII. Cell Signal. 2003; 15:1049-1057.

23. Previdi S, Abbadessa G, Dalo F, France DS, Broggini M. Breast cancer-derived bone metastasis can be effectively reduced through specific c-MET inhibitor tivantinib
(ARQ 197) and shRNA c-MET knockdown. MolCancer Ther. 2012; 11:214-223.

24. Pennacchietti S, Michieli P, Galluzzo M, Mazzone M, Giordano S, Comoglio PM. Hypoxia promotes invasive growth by transcriptional activation of the met protooncogene. Cancer cell. 2003; 3:347-361.

25. Jahangiri A, De Lay M, Miller LM, Carbonell WS, $\mathrm{Hu}$ YL, Lu K, Tom MW, Paquette J, Tokuyasu TA, Tsao S, Marshall R, Perry A, Bjorgan KM, Chaumeil MM, Ronen SM, Bergers G, et al. Gene expression profile identifies tyrosine kinase c-Met as a targetable mediator of antiangiogenic therapy resistance. Clinical cancer research: an official journal of the American Association for Cancer Research. 2013; 19:1773-1783.

26. Wertz IE, Kusam S, Lam C, Okamoto T, Sandoval W, Anderson DJ, Helgason E, Ernst JA, Eby M, Liu J, Belmont LD, Kaminker JS, O'Rourke KM, Pujara K, Kohli PB, Johnson AR, et al. Sensitivity to antitubulin chemotherapeutics is regulated by MCL1 and FBW7. Nature. 2011; 471:110-114.

27. Nicoletti I, Migliorati G, Pagliacci MC, Grignani F, Riccardi C. A rapid and simple method for measuring thymocyte apoptosis by propidium iodide staining and flow cytometry. JImmunolMethods. 1991; 139:271-279. 